\title{
A 4-41 GHz Singly Balanced Distributed Mixer Using GaAs pHEMT Technology
}

\author{
Fong-Cheng Chang, Pei-Si Wu, Student Member, IEEE, Ming-Fong Lei, Student Member, IEEE, and
} Huei Wang, Fellow, IEEE

\begin{abstract}
A broadband singly balanced distributed mixer is developed using a $0.15-\mu \mathrm{m}$ GaAs pHEMT foundry process. It is the first time that the charge-injection approach is applied to a distributed mixer. With the advantage of charge-injection, the mixer achieves a high conversion gain with low dc consumption. The fabricated distributed mixer with an integrated broadband transformer has a compact chip size of $2 \mathrm{~mm} \times 1 \mathrm{~mm}$. Measurement results show that the mixer achieves a conversion gain of better than $3.5 \mathrm{~dB}$ over a broadband frequency from $4-41 \mathrm{GHz}$, with a relatively low de power consumption of $100 \mathrm{~mW}$.
\end{abstract}

Index Terms-Active mixer, broadband mixer, distributed circuit, down-conversion, GaAs monolithic, mixer, monolithic microwave integrated circuit (MMIC).

\section{INTRODUCTION}

$\mathbf{T}$ HE increasing bandwidth demands in broadband systems and wireless communication systems drive integrated circuit design to operate at higher frequencies. So far, the concept of distributed circuits has offered attractive solutions to some challenging problems in high speed communication systems. The operation principle of distributed circuits is based on multiple parallel signal paths working with in-phase combination that can be used to extend the frequency of operation. The artificial transmission line approach may be adopted, which absorbs parasitic capacitances of active device into the artificial transmission lines [1].

Various types of distributed mixers using all kinds of MMIC technologies were implemented with impressive performance and high operation frequency. A dc to 38-GHz InP HEMT distributed mixer was presented with a conversion loss of $5 \mathrm{~dB}$ [2]. A CMOS distributed mixer has been published with a conversion gain of $3 \mathrm{~dB}$ over a bandwidth from 3 to $8.72 \mathrm{GHz}$ [3]. Recently, a GaAs distributed drain mixer has been demonstrated from 3 to $33 \mathrm{GHz}$ which shows a conversion loss of $4 \mathrm{~dB}$ [4]. Another GaAs distributed mixer with cascode FET cells shows a high conversion gain of $3.6 \mathrm{~dB}$ over a wide bandwidth from 3 to $40 \mathrm{GHz}$, but with a high dc power consumption [5].

In this letter, we propose a distributed mixer using singly balanced mixers with charge-injected transistors as unit sections

Manuscript received August 22, 2006; revised September 27, 2006. This work was supported by the National Science Council of Taiwan., R.O.C., under Projects NSC 93-2219-E-002-024, NSC 93-2213-E-002-033, and NSC 93-2752-E-002-002-PAE and by WIN Semiconductors, Taoyuan, Taiwan, R.O.C., through the Chip Implementation Center, Hsinchu, Taiwan, R.O.C.

The authors are with the Department of Electrical Engineering and Graduate Institute of Communication Engineering, National Taiwan University, Taipei 106, Taiwan, R.O.C. (e-mail: hueiwang@ew.ee.ntu.edu.tw).

Color versions of one or more of the figures in this letter are available online at http://ieeexplore.ieee.org.

Digital Object Identifier 10.1109/LMWC.2006.890341

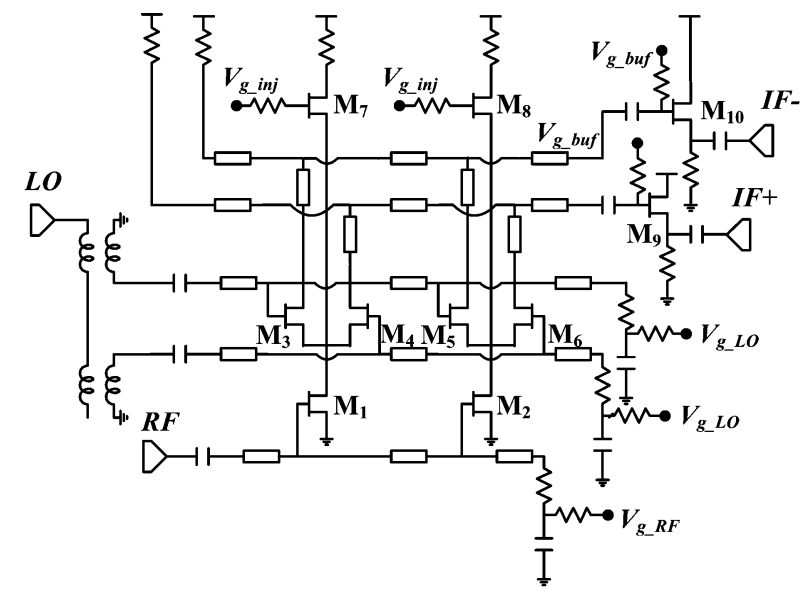

Fig. 1. Circuit schematic of the proposed distributed mixer.

to build a two-stage distributed mixer. The MMIC mixer was fabricated using commercial $0.15-\mu \mathrm{m}$ GaAs pHEMT foundry process. The measured results show that the conversion gain of the mixer is better than $3.5 \mathrm{~dB}$ from 4 to $41 \mathrm{GHz}$ with IF frequency fixed at $1 \mathrm{GHz}$. By applying charge-injection technique [6], the mixer achieves high conversion gain at a low dc power level, which rivals the published results to date.

\section{Circuit Design And Fabrication}

The schematic of the proposed distributed mixer composing two identical sections is shown in Fig. 1. The distributed mixer is based on a singly balanced mixer core. The input capacitances of the local oscillator (LO) differential-pair and the radio frequency (RF) transistors are absorbed into the artificial transmission lines. Therefore, the input impedance remains constant until the cutoff frequency of the artificial transmission line. The artificial transmission line can be considered as a $L C$ ladder network where the inductors are implemented with microstrip lines on the GaAs substrate, and capacitances are the parasitic capacitances, specifically, $C_{\mathrm{gs}}$ and $C_{\mathrm{ds}}$ of the transistors. The termination load connected at the end of the artificial transmission line was designed to match the characteristic impedance of the artificial transmission lines to reduce the voltage standing wave ratio (VSWR). As the RF signal travels from the input port and propagates through the RF artificial transmission line, the RF voltage sweeps across the gate-source capacitances of the transistors producing a current proportional to the RF signal which propagates toward the LO differential-pair transistors. The LO differential-pairs were driven by the LO power to modulate the current produced by RF signal. 


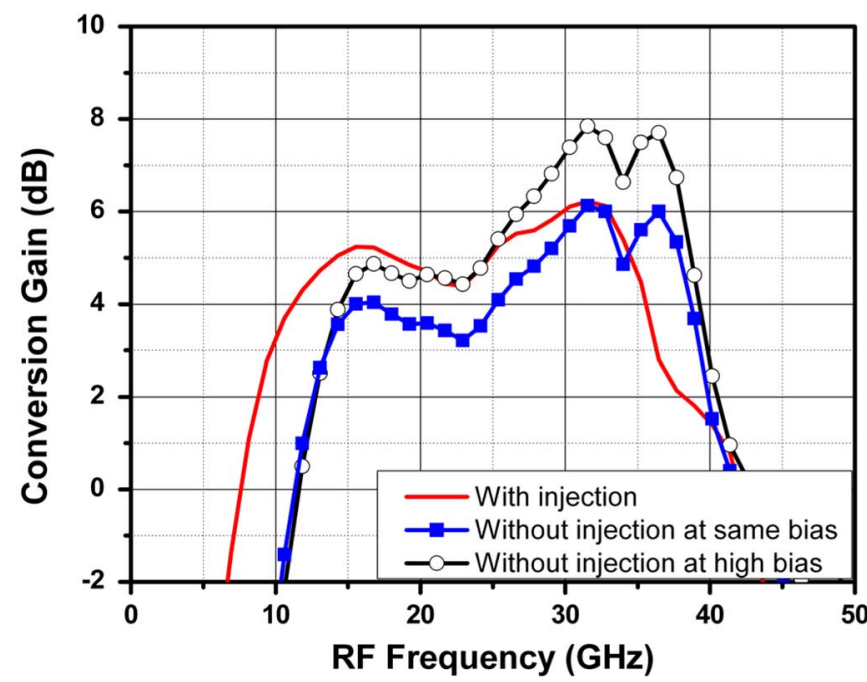

Fig. 2. Simulated results of the effect of the charge-injection technique.

Due to its smaller parasitic capacitance, transistors with a gate periphery of $100 \mu \mathrm{m}$ were selected to achieve wideband performance. To obtain a pair of high quality LO signals, we adopted a broadband Marchand-type transformer to convert the single-ended signal to differential signal conversion [7]. The transformer occupies a small size of $0.14 \mathrm{~mm} \times 0.28 \mathrm{~mm}$ and shows a simulated performance with amplitude imbalance and phase imbalance smaller than $2 \mathrm{~dB}$ and $5^{\circ}$ from 10 to $40 \mathrm{GHz}$, respectively.

The charge-injection technique was applied for the distributed mixer biasing. The charge-injection transistors are $M_{7-8}$ in Fig. 1. To avoid the additional dc power dissipation in the LO differential-pair and to relax the voltage headroom of load resistors, another current path is provided at the drain of RF transistors $\left(M_{1-2}\right)$ to inject additional biasing current. In addition to reducing the dc power dissipation, the conversion gain also can be increased by injecting more biasing current to the RF transistors. The effect of charge-injection is also investigated. Fig. 2 shows the simulated results of mixer with and without charge-injection. If the bias conditions of both mixers (with and without charge-injection) are the same, the conversion gain of the mixer with charge-injection is $1-2 \mathrm{~dB}$ higher than the mixer without charge-injection. To achieve comparable performance, the mixer without charge-injection needs to be biased higher and dissipates $25 \mathrm{~mW}$ more than the mixer with charge-injection. The down-converted IF signal power goes through a buffer amplifier with an estimated 5-dB gain to the output. Fig. 3 shows the chip micrograph with a compact size of $2 \mathrm{~mm} \times 1 \mathrm{~mm}$.

\section{Circuit Performance}

The chips were measured using on-wafer probing on RF and LO input ports with ground-signal-ground (GSG) probes, and the output IF signal ports were measured using GSSG probes. One of the IF outputs was terminated to a $50-\Omega$ load, and the other one was connected to a spectrum analyzer. All power levels are corrected to the circuit's input and output probes. The single-ended IF output was measured and a 3-dB correction factor was added for the differential conversion gain. The

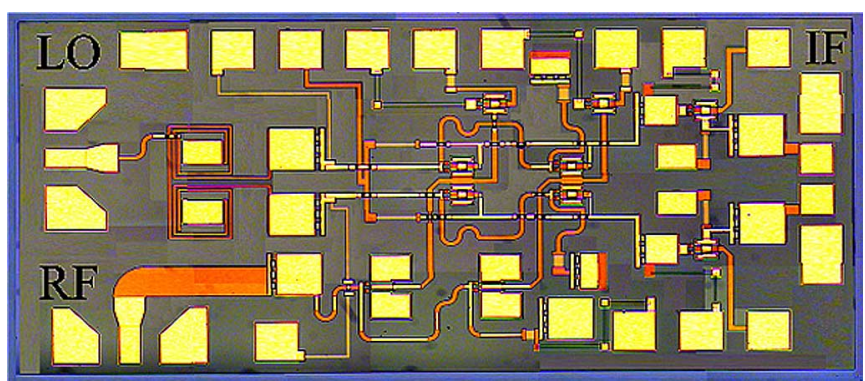

Fig. 3. Chip photograph of the proposed distributed mixer. The chip size is $2 \mathrm{~mm} \times 1 \mathrm{~mm}$.

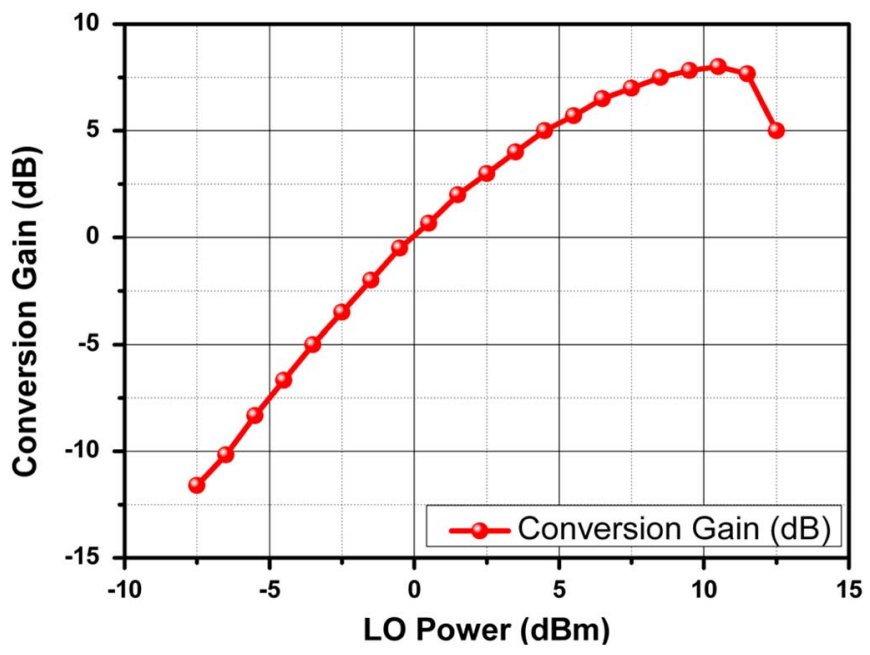

Fig. 4. Measured LO power versus conversion gain, of which RF frequency is fixed at $20 \mathrm{GHz}$, and IF bandwidth is $1 \mathrm{GHz}$.

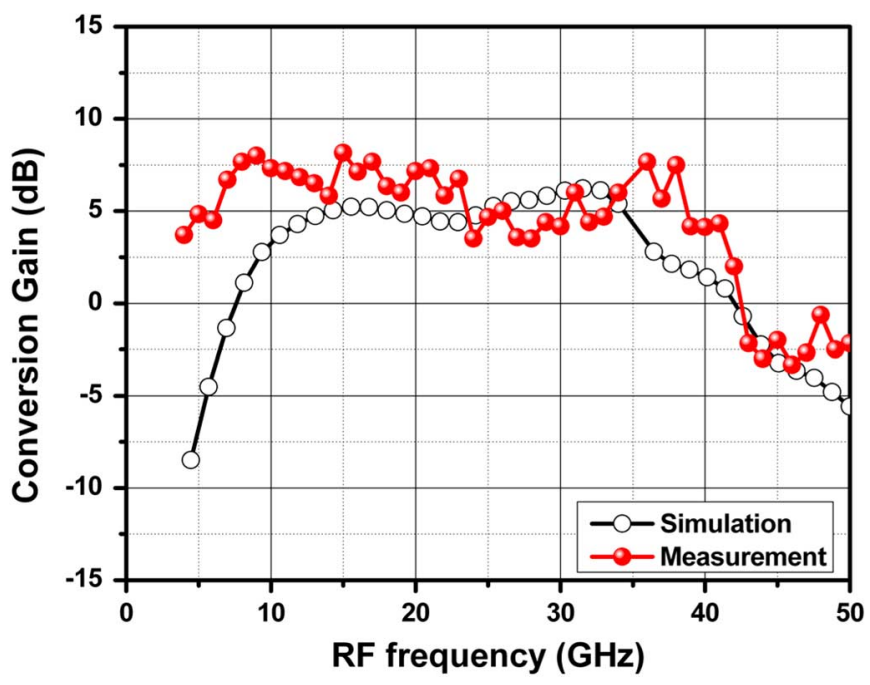

Fig. 5. Simulated and measured conversion gain versus RF frequency, of which LO power is $10 \mathrm{dBm}$ and IF frequency is fixed at $1 \mathrm{GHz}$.

measured conversion gain swept with $\mathrm{LO}$ power saturated at an LO power level of $10 \mathrm{dBm}$ at RF frequency of $20 \mathrm{GHz}$ and IF frequency of $1 \mathrm{GHz}$, as shown in Fig. 4. The conversion gain versus the RF frequency is shown in Fig. 5, with both RF and LO frequency sweeping form 4 to $50 \mathrm{GHz}$, a fixed IF frequency of $1 \mathrm{GHz}$, and $\mathrm{LO}$ power of $10 \mathrm{dBm}$. A high average conversion gain of $5.8 \mathrm{~dB}$ over a broadband frequency from 4 to $41 \mathrm{GHz}$ is 
TABLE I

Summary of THE Previously Published MMIC Distributed Mixers AND THIS WORK

\begin{tabular}{|c|c|c|c|c|c|c|c|}
\hline Ref. & $\begin{array}{l}\text { Freq. } \\
(\mathrm{GHz})\end{array}$ & Process & Unit cell & $\begin{array}{c}\text { Conversion } \\
\text { Gain } \\
(\mathrm{dB}) \\
\end{array}$ & $\begin{array}{c}\text { LO-RF } \\
\text { Isolation } \\
(\mathrm{dB})\end{array}$ & $\begin{array}{c}\text { Power } \\
\text { Dissipation } \\
(\mathrm{mW})\end{array}$ & $\begin{array}{c}\text { Chip Size } \\
(\mathrm{mm} \times \mathrm{mm})\end{array}$ \\
\hline [2] & DC-38 & InP HEMT & Gilbert cell & -5 & - & 2500 & $2 \times 2$ \\
\hline [3] & $3-8.7$ & $0.18 \mu \mathrm{m}$ CMOS & Singly balanced mixer & $2.5 \sim 5$ & - & 10.8 & $1.4 \times 1.2$ \\
\hline [4] & $3-33$ & $0.15 \mu \mathrm{m}$ GaAs pHEMT & Single device & $-1 \sim-4$ & 20 & 77 & $1.7 \times 1$ \\
\hline [5] & $3-40$ & $0.15 \mu \mathrm{m}$ GaAs pHEMT & Cascode device & 3.6 & 19 & 200 & $1.8 \times 1$ \\
\hline [8] & $5-39$ & $0.15 \mu \mathrm{m}$ GaAs pHEMT & Tri-cascode device & $-2 \sim-5$ & - & 1100 & $3 \times 1.1$ \\
\hline [9] & $10-50$ & $0.2 \mu \mathrm{m}$ GaAs pHEMT & Single device & -3 & - & 240 & $4 \times 1$ \\
\hline $\begin{array}{l}\text { This } \\
\text { Work }\end{array}$ & $4-41$ & $0.15 \mu \mathrm{m}$ GaAs pHEMT & Singly balanced mixer & $\begin{array}{c}3.5 \sim 8^{*} \\
\text { (with buffer) }\end{array}$ & 19 & 100 & $2 \times 1$ \\
\hline
\end{tabular}

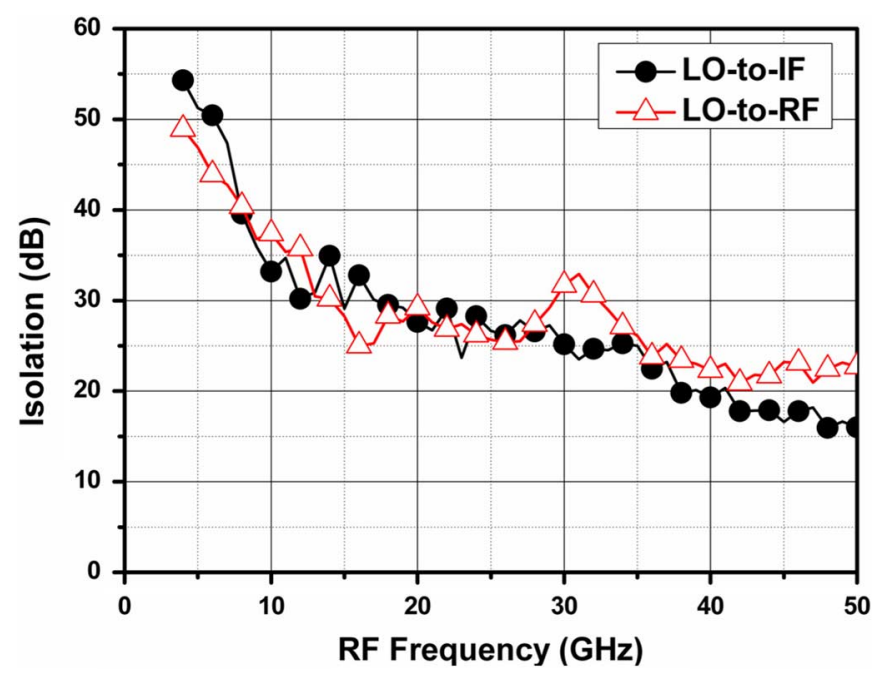

Fig. 6. Measured results of isolations versus RF frequency, of which LO power is $10 \mathrm{dBm}$ and $\mathrm{IF}$ frequency is fixed at $1 \mathrm{GHz}$.

achieved. With the advantages of charge-injection, it dissipates a relatively low dc power of $100 \mathrm{~mW}$, compared with the performance of previously reported mixers.

The measured LO-to-RF and LO-to-IF isolation is shown in Fig. 6. The measured LO-to-IF and LO-to-RF isolation are better than 19 and $22 \mathrm{~dB}$ from 4 to $41 \mathrm{GHz}$, respectively.

Table I summarizes the previous results and this work [8], [9]. This mixer demonstrates broadband performance at a relatively low dc power of $100 \mathrm{~mW}$ from 4 to $41 \mathrm{GHz}$. LO-to-RF and LO-to-IF isolation are all better than $19 \mathrm{~dB}$ from 4 to $41 \mathrm{GHz}$.

\section{CONCLUSION}

A distributed mixer using the GaAs pHEMT process is presented. With the advantage of charge-injection, the mixer achieved a high average conversion gain of $5.8 \mathrm{~dB}$ at a power dissipation of $100 \mathrm{~mW}$ over a very wideband frequency, which outperforms previously published distributed mixers.

\section{ACKNOWLEDGMENT}

The authors would like to thank Dr. H.-Y. Chang, National Central University, for his helpful suggestions.

\section{REFERENCES}

[1] O. S. A. Tang and C. S. Aitchison, "A very wide-band microwave MESFET mixer using the distributed mixing principle," IEEE Trans. Microw. Theory Tech., vol. MTT-33, no. 12, pp. 1470-1478, Dec. 1985.

[2] Y. Imai, S. Kimura, Y. Umeda, and T. Enoki, "A DC to 38-GHz distributed analog multiplier using InP HEMT's," IEEE Microw. Guided Wave Lett., vol. 4, no. 12, pp. 399-401, Dec. 1994.

[3] A. Q. Safarian, A. Yazdi, and P. Heydari, "Design and analysis of an ultrawide-band distributed CMOS mixer," IEEE Trans. VLSI Syst., vol. 13, no. 5, pp. 1470-1478, May 2005.

[4] K. L. Deng and H. Wang, "A 3-33 GHz PHEMT mmic distributed drain mixer," in IEEE RFIC Symp. Dig., Jun. 2002, pp. 151-154.

[5] W. Ko and Y. Kwon, "A GaAs-based 3-40 GHz distributed mixer with cascade FET cells," in Proc. IEEE Rad. Freq. Integr. Circuits Symp., Jun. 2004, pp. 413-416.

[6] L. A. MacEachem and T. Manku, "A charge-injection method for Gilbert cell biasing," in Proc. IEEE Canadian Conf. Elect. Comp. Eng., May 1998, vol. 1, pp. 265-368.

[7] P.-S. Wu, C.-H. Wang, T.-W. Huang, and H. Wang, "Compact and broad-band millimeter-wave monolithic transformer balanced mixers," IEEE Trans. Microw. Theory Tech., vol. 53, no. 10, pp. 3106-3114, Oct. 2005.

[8] I. Kallfass, T. Purtova, A. Brokmeier, W. Ludwig, and H. Schumacher, "One single traveling-wave MMIC for highly linear broadband mixer and variable gain amplifiers," in IEEE MTT-S Int. Dig., Jun. 2005, pp. 97-100.

[9] D. Hollmann, R. Heilig, and G. Baumann, "A monolithic broadband 10-50 GHz distributed HEMT mixer including active LO-RF combiner," in IEEE GaAs IC Symp. Dig., Oct. 1994, pp. 100-103. 